\title{
Development of matrix solid-phase dispersion method for the extraction of short-chain chlorinated paraffins in human placenta
}

\author{
Ying Wang ${ }^{1,2}$, Wei Gao ${ }^{1,3}$, Jing $\mathrm{Wu}^{1,3}$, Huijin $\mathrm{Liu}^{2, *}$, Yingjun Wang ${ }^{1,3}$, \\ Yawei Wang ${ }^{1,3,4, *}$, Guibin Jiang ${ }^{1,4}$
}

1. State Key Laboratory of Environmental Chemistry and Ecotoxicology, Research Center for Eco-Environmental Sciences, Chinese Academy of Sciences, Beijing 100085, China. E-mail: 372907737@qq.com

2. College of Chemistry and Chemical Engineering, Gannan Normal University, Ganzhou 341000, China

3. University of Chinese Academy of Sciences, Beijing 100049, China

4. Institute of Environment and Health, Jianghan University, Wuhan 430056, China

\section{A R T I C L E I N F O}

\section{Article history:}

Received 24 January 2017

Revised 12 June 2017

Accepted 29 June 2017

Available online 11 July 2017

Keywords:

Short-chain chlorinated paraffins

Matrix solid-phase dispersion

Human placenta

\begin{abstract}
A B S T R A C T
Chlorinated paraffins (SCCPs) are widely used worldwide, and they can be released into the environment during their production, transport, usage and disposal, which pose potential risks for human health. In this work, an efficient, reliable and rapid pretreatment method based on matrix solid-phase dispersion (MSPD) was developed for the analysis of short-chain CPs (SCCPs) in human placenta by gas chromatograph-electron capture negative ion low-resolution mass spectrometry (GC-ECNI-LRMS) and gas chromatography-quadrupole time-of-flight mass spectrometry (GC-QTOF-HRMS). The MSPD-relevant parameters including dispersing sorbent, sample-to-sorbent mass ratio, and elution solvent were optimized using the orthogonal test. Silica gel was found to be the optimal dispersing sorbent among the selected matrices. Under the optimal conditions, $44 \%$ acidic silica gel can be used as the co-sorbent to remove lipid and eluted by the mixture of hexane and dichloromethane $(7: 3, \mathrm{~V} / \mathrm{V})$. The spiked recoveries of the optimized method were $77.4 \%$ and $91.4 \%$ for analyzing SCCPs in human placenta by GC-ECNI-LRMS and GC-QTOF-HRMS, and the corresponding relative standard deviations were $10.2 \%$ and 5.6\%, respectively. The method detection limit for the total SCCPs was $36.8 \mathrm{ng} / \mathrm{g}$ (dry weight, dw) and $19.2 \mathrm{ng} / \mathrm{g}(\mathrm{dw})$ as measured by GC-ECNI-LRMS and GC-QTOF-HRMS, respectively. The concentrations of SCCPs in four human placentas were in the range of $<$ method detection limit (MDL) to $782 \mathrm{ng} / \mathrm{g}(\mathrm{dw})$, which is also the first description of SCCPs detected in placentas.
\end{abstract}

(c) 2017 The Research Center for Eco-Environmental Sciences, Chinese Academy of Sciences. Published by Elsevier B.V.

\section{Introduction}

Short-chain chlorinated paraffins (SCCPs) are highly complex technical mixtures of polychlorinated $n$-alkanes. SCCPs can be released into the environment during production, storage, transportation and product use as fire retardants and plasticizers in polyvinyl chloride (PVC), rubber, other plastics, varnishes, sealants, metal-cutting oils, etc. (Campbell and

\footnotetext{
* Corresponding authors. E-mails: chemlhj@163.com (Huijin Liu), ywwang@rcees.ac.cn (Yawei Wang).
} 
McConnell, 1980). SCCPs have been found in a variety of environmental matrices, such as water (Coelhan, 2010), soil and sediment (Chen et al., 2011; Gao et al., 2012; Zeng et al., 2013), air (Barber et al., 2005; Wang et al., 2013), dust (Fridén et al., 2011), food (Harada et al., 2011; Iino et al., 2005), a quatic biota (Bennie et al., 2000; Jansson et al., 1993; Tomy et al., 2000), and breast milk (Thomas et al., 2006). Due to their persistence, toxicological properties, capability to bioaccumulate and potential long-range air transport, SCCPs have aroused wide attention. In 2006, the European Union submitted a proposal to list SCCPs under the Stockholm Convention (SC) as Persistent Organic Pollutants (POPs). After ten years, the eighth Conference of Parties finally decided to list SCCPs in Annex A in 2017 of the SC.

At different stages of human development, toxic chemicals can enter the human body via different pathways which embody placenta transport such as fetuses, breast milk to infants, and food as children and adults (Cheng et al., 2015). Like other POPs (e.g., dioxins, polychlorinated biphenyls (PCBs) and organochlorine pesticides), SCCPs can cause toxicological effects in mammals and may affect the liver, thyroid hormone system, and kidneys, e.g., by causing hepatic enzyme induction and thyroid hyperactivity, further leading to carcinogenicity in these organs (UNEP/POPRC.11/10/Add.2, 2015). SCCPs may lead to adverse effects for human health, especially for fetuses that have weak defense mechanisms to toxicant. The pre- and neo-natal periods are the most crucial periods of individual growth. During these periods the organs are developing gradually, and the defense mechanisms against toxic substance are poorly developed. Thus, the effect of such intake of toxic substances can be especially detrimental and permanent, which have been proved about the adverse effects of prenatal exposure to various POPs (e.g., polycyclic aromatic hydrocarbons (PAHs), PCBs, polychlorinated dibenzop-dioxins (PCDDs), polychlorinated dibenzofurans (PCDFs), polybrominated diphenyl ethers (PBDEs), and dechlorane plus (DP)) on fetal development (Ben et al., 2014; Dewailly et al., 1993; Huel et al., 1992; Main et al., 2007).

The placenta is an ephemeral organ that grows with the developing fetus. Although the placenta acts as a barrier and transports nutrients and oxygen to the fetus, many toxic compounds can be transported across the placenta to some degree and therefore influence the unborn child (Myren et al., 2007). Therefore, the chemical concentration at the time of child delivery may indicate the burden for both the mother and neonate and reflect the levels of exposure during the entire pregnancy. However, to our knowledge, no investigation on prenatal exposure to SCCPs has been performed due to the lack of methods for analyzing SCCPs in the placenta.

Matrix solid-phase dispersion (MSPD) is a simple, rapid and efficient method of sample preparation for complex matrices, and it has been applied to pretreat solid, semisolid and highly viscous and toughened samples. The method does not require special instruments and it allows simultaneous sample dispersion, extraction and cleanup in one single step. The MSPD procedure consists of blending the matrix onto a solid sorbent, allowing matrix cell disruption and subsequently extracting the target analytes by means of a suitable elution solvent (Barker, 2000;
Capriotti et al., 2010). The MSPD extraction technique has been successfully applied to the trace analysis of POPs from biological matrixes such as PCBs from animal fatty samples (Criado et al., 2004), pesticides, PCBs, PBDEs and polybrominated biphenyl (PBBs) from several marine species (Carro et al., 2005), and PAHs from fish tissue (Pensado et al., 2005).

In this work, we aimed to develop a simple, rapid, efficient, and economic method based on MSPD to analyze SCCPs in human placenta by gas chromatograph-electron capture negative ion low-resolution mass spectrometry (GC-ECNI-LRMS) and gas chromatography-quadrupole time-of-flight highresolution mass spectrometry (GC-QTOF-HRMS). Three factors (dispersing sorbent, sample-to-sorbent mass ratio and elution solvent) are critical to the MSPD extraction performance for SCCPs, and they were optimized by using a three-factor, four-level orthogonal test. The final proposed method was applied to real human placenta samples. To our knowledge, this is the first study on the analytical method of placenta samples, which can help us to investigate the potential internal exposure of SCCPs of pregnant woman, further to assess the potential risk to the fetus by CPs.

\section{Materials and methods}

\subsection{Chemicals and reagents}

The SCCP mixture standards $\left(\mathrm{C}_{10}-\mathrm{C}_{13}\right.$, chlorine contents of $51.5 \%, 55.5 \%, 63.0 \%, 100 \%$ purity, $100 \mathrm{ng} / \mu \mathrm{L})$ and $\varepsilon$-hexachlorocyclohexane $(\varepsilon-\mathrm{HCH}, 99.9 \%$ purity, $10 \mathrm{ng} / \mu \mathrm{L})$ were purchased from Dr. Ehrenstorfer GmbH (Augsburg, Germany). The 1,5,5,6,6,10-Hexachlorodecane $\left({ }^{13} \mathrm{C}_{10^{-}}, 100 \mathrm{ng} / \mu \mathrm{L}\right.$ solution in cyclohexane) was obtained from Cambridge Isotope Laboratories (USA). Cyclohexane, dichloromethane (DCM), hexane, and acetone for the pesticide residue analysis were obtained from J.T. Baker (USA). Sulfuric acid and anhydrous sodium sulfate were all guaranteed reagents purchased from Sinopharm Chemical Reagent Beijing Co., Ltd. (China). Bondesil-C 8 $(40 \mu \mathrm{m})$, and Bondesil- $\mathrm{C}_{18}(40 \mu \mathrm{m})$ were purchased from Agilent Technologies (USA). Silica gel $(0.063-0.100 \mathrm{~mm})$ was obtained from Merck KGaA (Darmstadt, Germany). Florisil (60-100 meshes) was obtained from Supelco (Bellefonte, PA). Before use, anhydrous sodium sulfate and silica gel were heated to 650 and $550^{\circ} \mathrm{C}$, respectively, in a muffle furnace for $10 \mathrm{hr}$. Florisil was heated to $140^{\circ} \mathrm{C}$ in a muffle furnace for $7 \mathrm{hr}$.

\subsection{Sample preparation}

Four placenta samples were collected from a hospital and then stored in a freezer at $-20^{\circ} \mathrm{C}$. The procedures were approved by the Ethic Committee of Research Center for Eco-Environmental Sciences and were in compliance with research requirements regarding human subjects. Before extraction, the excess blood from the placenta was washed away with Milli-Q pure water, and the connective tissues were also removed. Then, the placenta was cut into small pieces, freeze-dried, homogenized in a pulverizer, and packed tightly with aluminum foil and a valve bag. 
Because the placenta sample is peculiar and hard to obtain, we used fish muscle to represent the placenta sample during the optimization of the extraction procedure, and the final method was validated by analyzing real placenta samples spiked by an SCCP standard. Before extraction, the fish sample was prepared in the same manner as the placenta as described above.

The extraction efficiency was evaluated by the matrixspiked recoveries of the analytes, and the samples were spiked with standards as follows (Liu et al., 2011): a certain amount of SCCP standard $\left(\mathrm{C}_{10}-\mathrm{C}_{13}\right.$, chlorine contents of $\left.55.5 \%\right)$ was spiked into the dried samples and then homogenized in a mortar and covered with aluminum foil in a refrigerator at $4^{\circ} \mathrm{C}$ overnight to allow sufficient dispersion and equilibration.

\subsection{General MSPD extraction procedure}

The MSPD procedure is described as follows: $1 \mathrm{~g}$ freeze-dried sample and $1 \mathrm{~g}$ dispersing sorbent were weighed into a glass mortar. The glass mortar was washed with acetone, DCM, and hexane in sequence before use. After adding $10 \mathrm{ng}$ of surrogate standard $\left({ }^{13} \mathrm{C}_{10}-1,5,5,6,6,10\right.$-hexachlorodecane) and $5 \mathrm{~g}$ of anhydrous sodium sulfate, the sample-sorbent mixture was thoroughly ground using a glass pestle. Then, the blend was transferred to a glass column $(15 \mathrm{~mm}$ id and $30 \mathrm{~cm}$ in length) with Polytetrafluoroethylene (PTFE) cocks pre-packed with $4 \mathrm{~g}$ of anhydrous sodium sulfate and $5 \mathrm{~g}$ of acid silica gel (W/W, $44 \% \mathrm{H}_{2} \mathrm{SO}_{4}$ ). Afterwards, $3 \mathrm{~g}$ of anhydrous sodium sulfate was packed at the top of the glass column. The column was eluted by $100 \mathrm{~mL}$ of the mixture of hexane and $\operatorname{DCM}(7: 3, \mathrm{~V} / \mathrm{V})$ and approximately $40 \mathrm{~mL}$ of the elution solvent was used to rinse the mortar and pestle three times before use. The elution was concentrated to approximately $2 \mathrm{~mL}$ by the rotary evaporator and thereafter concentrated to near-dryness under a gentle nitrogen flow. Then, the solvent was changed to a final volume of $200 \mu \mathrm{L}$ cyclohexane in a minivial. Finally, prior to instrument analysis, the recovery standard of $\varepsilon-\mathrm{HCH}$ (10 ng) was added in the vial and mixed by vortexing.

\subsection{Instrument analysis and quantification}

GC-ECNI-LRMS has been used to analyze SCCPs in various environmental samples in previous studies. In this work, in order to make a contrast, a novel analytical approach based on GC-QTOF-HRMS was also used to analyze SCCPs.

\subsubsection{GC-ECNI-LRMS}

The instrument analysis method for SCCPs by GC-ECNI-LRMS was described in detail in our previous paper (Wang et al., 2012; Zeng et al., 2011). Briefly, SCCPs were determined by an Agilent 7890A GC system coupled to an Agilent 7000 Triple Quadrupole MS and a 7683B series injector. GC separations were performed using a DB-5 ms (30 length, $0.25 \mathrm{~mm}$ i.d., $0.25 \mu \mathrm{m}$ film thickness) capillary column. Helium (99.999\%) was used as the carrier gas at a flow rate of $1.0 \mathrm{~mL} / \mathrm{min}$, and methane was the reagent gas. The final extract $(1 \mu \mathrm{L})$ was injected in pulse splitless mode. The injector temperature, transfer line temperature and ion source temperature were set to 280,280 , and $200^{\circ} \mathrm{C}$, respectively. The GC oven temperature gradient was as follows: initial temperature at $100^{\circ} \mathrm{C}$ (held for $1 \mathrm{~min}$ ), then increased to $160^{\circ} \mathrm{C}$ at $30^{\circ} \mathrm{C} / \mathrm{min}$ (held for $5 \mathrm{~min}$ ), and finally increased to $310^{\circ} \mathrm{C}$ at $30^{\circ} \mathrm{C} / \mathrm{min}$ (held for $12 \mathrm{~min}$ ). The date analysis was performed by Mass Hunter workstation software B.07. The quantification of 24 different SCCP formula groups $\left(\mathrm{C}_{10}-\mathrm{C}_{13}\right.$ with $5-10$ chlorine atoms) was conducted by four separate injections under selected monitoring (SIM) mode with dwell times of $50 \mathrm{~ms}$. The highest and sub-high abundant isotope ions of $[\mathrm{M}-\mathrm{Cl}]^{-}$were applied to quantification and confirmation, respectively, for which the $\mathrm{m} / \mathrm{z}$ values have been described in detail elsewhere (Reth and Oehme, 2004). The identification of the SCCP congener groups in the samples relied on the retention time and signal shape and on combining the calculation of the ratio of quantitative and qualitative ions based on the chromatographic and mass spectrometric results to minimize interference with similar nominal masses to SCCPs (Reth et al., 2005; Zeng et al., 2011).

\subsubsection{GC-QTOF-HRMS}

The data analysis method for SCCPs in environmental samples by GC-QTOF-HRMS (Agilent 7200, Santa, Clara, USA) was also reported previously in our laboratory (Gao et al., 2016). GC parameters were the same as the method of GC-ECNI-LRMS. The source temperature was set to $150^{\circ} \mathrm{C}$. The data was recorded by Mass Hunter Acquisition B.07, and full-scan mode was used in negative chemical ionization (NCI). SCCPs and medium-chain chlorinated paraffins (MCCPs) can be analyzed simultaneously without mutual interferences in a single injection by extracting accurate masses (accurate to four decimal places), the retention time and the signal shape matching the standards. The accurate $\mathrm{m} / \mathrm{z}$ values of the quantitative and qualitative $[\mathrm{M}-\mathrm{Cl}]^{-}$ions for SCCPs and the corresponding retention time for the 24 SCCP congener groups were also described in detail in our previous paper (Gao et al., 2016). Auto-integration was employed using Mass Hunter Quantitative Analysis B.07, whereas considering the interference from similar nominal mass, manual integration was used in the analytical method of GC-ECNI-LRMS.

\subsection{Quality control}

To avoid the possible contamination, all glassware was baked at $450^{\circ} \mathrm{C}$ after washing with Decon 90 (a lotion for organic matter) and deionized water in order and then washed with DCM and hexane three times prior to use. Procedural blanks were included in each batch of six samples. Ten nanograms of ${ }^{13} \mathrm{C}_{10}-1,5,5,6,6,10$-hexachlorodecane was added into all of the samples as a surrogate standard before extraction. All analyses were performed in triplicate. Moreover, for quantification, an 8-point calibration curve of SCCPs with different chlorine contents $(51.5 \%, 52.5 \%, 53.5 \%, 54.5 \%, 55.5 \%, 57.375 \%$, $59.25 \%$, and $63.0 \%$ ), all at $10 \mathrm{ng} / \mu \mathrm{L}$, was fitted between the total response factor of the set of SCCP standards (y-axis) and their calculated chlorine content ( $x$-axis), and 8 different chlorinated SCCP mixtures were obtained by mixing the SCCP standards with $51.5 \%, 55.5 \%$, and $63.0 \%$ chlorine, which has been described in detail elsewhere (Reth et al., 2005). Good linearity was obtained for both calibration curves as determined by GC-ECNI-LRMS $\left(R^{2}=0.980\right)$ and GC-QTOF-HRMS 
$\left(R^{2}=0.962\right)$, which were comparable with the previous report (Gao et al., 2016; Reth et al., 2005).

\section{Results and discussion}

\subsection{Optimization of the MSPD procedure}

The optimization of the MSPD procedure was performed with fish samples spiked with the $1000 \mathrm{ng}$ SCCP standard $\left(\mathrm{C}_{10}-\mathrm{C}_{13}\right.$, chlorine contents of 55.5\%) and non-spiked fish simultaneously. Primarily, three vital factors including dispersing sorbent (A), sample-to-sorbent mass ratio (B), and elution solvent (C) for the MSPD extraction efficiency were investigated by orthogonal experimental design. Four levels were set for each factor. Four dispersing sorbents, Florisil $\left(A_{1}\right)$, silica gel $\left(A_{2}\right), C_{18}\left(A_{3}\right)$, and $C_{8}\left(A_{4}\right)$, were examined in the investigation, and they are used extensively as the solid support in the MSPD method (Kristenson et al., 2006). Four ratios, 1:1 ( $\left.B_{1}\right), 1: 2$ $\left(B_{2}\right), 1: 3\left(B_{3}\right)$, and 1:4 $\left(B_{4}\right)$, were set for the sample-to-sorbent ratio (dry weight) which are typical ratios for the MSPD procedure (Kristenson et al., 2006). Considering the similar $\log K_{\text {ow }}$ between SCCPs (4.01-8.67) and other POPs, such as PCBs (4.61-7.67) (Bettina et al., 2011; Li et al., 2003), the mixture of hexane and DCM was chosen as the elution solvent, which is also always used for eluting PCBs during the cleanup procedure (Valsamaki et al., 2006), and the ratios of hexane: DCM were set as 1:1 $\left(C_{1}\right)$, 9:1 $\left(C_{2}\right), 8: 2\left(C_{3}\right)$, and 7:3 $\left(C_{4}\right),(V / V)$ respectively in this work. The final orthogonal experimental design $\mathrm{L}_{16}(4)^{5}$ is shown in Table 1 . The matrix-spiked recoveries of the 16 orthogonal experiments as determined by GC-ECNILRMS and GC-QTOF-HRMS are also presented in Table 1. The results indicate that the recoveries determined by GCQTOF-HRMS are higher than those by GC-ECNI-LRMS. The differences may be caused by the different sensitivity and the quantification method of the two instruments.
The range analysis aimed to clarify the levels of effect of different factors on the extraction efficiency. Thereby, the order of the effects of the three factors on the extraction efficiency can be known. The range analysis of the matrixspiked recoveries of the SCCPs is shown in Table 2. According to the $\mathrm{R}$ values determined by the two different instruments, the experimental factors can be ranked in the same order of the effect on the mean matrix-spiked recovery: elution solvent $(C)>$ dispersing sorbents $(A)>$ sample-to-sorbent ratio (B). The result indicated that among the three factors, the elution solvent was the most important factor affecting the extraction efficiency of SCCPs in the MSPD extraction procedure. Moreover, according to the highest mean recovery of each factor ( $T$ values), the optimum operating conditions determined by GC-ECNI-HRMS were selected as follows: $\mathrm{C}_{8}$ as the dispersing sorbent, $1: 3$ as the sample-to-sorbent mass ratio, and the 7:3 (V/V) mixture of normal hexane and DCM as the elution solvent. Similarly, the optimum operating conditions determined by GC-QTOF-HRMS were demonstrated to be as follows: $\mathrm{C}_{8}$ as the dispersing sorbent, 1:1 as the sample-to-sorbent mass ratio, and the 7:3 (V/V) mixture of normal hexane and DCM as the elution solvent. Although the optimum operating conditions determined by the two instruments are different on the sample-to-sorbent mass ratio, the sample-to-sorbent ratio is not the key factor for the extraction efficiency. Therefore, considering the cost and eluent velocity, the 1:1 sample-to-sorbent ratio was selected for the final pretreatment.

The effects of the interference removed in fish and placenta samples using $C_{8}$ and silica gel are shown in Fig. 1. Although the results of the orthogonal test certify that $C_{8}$ as the dispersing sorbent can attain the highest matrix-spiked recovery, it has some disadvantages, such as the higher cost and more interference. Compared with $\mathrm{C}_{8}$, silica gel (the second highest matrix-spiked recovery) can also attain a satisfactory recovery. Hence, finally we selected silica gel as the dispersing sorbent in this work due to its lower cost and less interference.

\section{Table 1 - Design of Orthogonal Table $\mathrm{L}_{16}(4)^{5}$ and the spiked recoveries of $\Sigma$ SCCPs in fish samples.}

\begin{tabular}{|c|c|c|c|c|c|}
\hline \multirow[t]{2}{*}{ Exp. no. } & \multirow{2}{*}{$\begin{array}{l}\text { (A)Dispersing } \\
\text { sorbent }\end{array}$} & \multirow{2}{*}{$\begin{array}{l}\text { (B)Sample: dispersing } \\
\text { sorbent }(\mathrm{m}: \mathrm{m})\end{array}$} & \multirow{2}{*}{$\begin{array}{c}\text { (C)Elution solvent } \\
\text { (hexane:dichloromethane, V/V) }\end{array}$} & \multicolumn{2}{|c|}{ Spiked recoveries $(\%)(n=3)$} \\
\hline & & & & GC-ECNI-LRMS & GC-QTOF-HRMS \\
\hline 1 & $\left(A_{1}\right)$ florisil & $\left(B_{1}\right) 1: 1$ & $\left(C_{4}\right) 7: 3$ & $82.1 \pm 0.6$ & $124.4 \pm 1.1$ \\
\hline 2 & florisil & $\left(B_{2}\right) 1: 2$ & $\left(C_{3}\right) 8: 2$ & $72.0 \pm 12.2$ & $124.3 \pm 3.7$ \\
\hline 3 & florisil & $\left(B_{3}\right) 1: 3$ & $\left(C_{1}\right) 1: 1$ & $89.6 \pm 2.3$ & $120.5 \pm 5.1$ \\
\hline 4 & florisil & $\left(B_{4}\right) 1: 4$ & $\left(C_{2}\right) 9: 1$ & $76.4 \pm 2.6$ & $98.6 \pm 3.7$ \\
\hline 5 & $\left(A_{2}\right)$ Silica gel & $1: 1$ & $1: 1$ & $90.5 \pm 5.8$ & $119.3 \pm 5.6$ \\
\hline 6 & Silica gel & $1: 2$ & $9: 1$ & $70.8 \pm 2.8$ & $105.5 \pm 1.8$ \\
\hline 7 & Silica gel & $1: 3$ & $7: 3$ & $86.2 \pm 1.3$ & $127.0 \pm 4.7$ \\
\hline 8 & Silica gel & $1: 4$ & $8: 2$ & $78.1 \pm 0.9$ & $117.6 \pm 2.7$ \\
\hline 9 & $\left(\mathrm{~A}_{3}\right) \mathrm{C}_{18}$ & $1: 1$ & $8: 2$ & $78.0 \pm 4.7$ & $115.1 \pm 1.6$ \\
\hline 10 & $\mathrm{C}_{18}$ & $1: 2$ & $7: 3$ & $83.1 \pm 1.1$ & $113.6 \pm 4.0$ \\
\hline 11 & $\mathrm{C}_{18}$ & $1: 3$ & $9: 1$ & $63.0 \pm 0.6$ & $102.8 \pm 5.2$ \\
\hline 12 & $\mathrm{C}_{18}$ & $1: 4$ & $1: 1$ & $74.2 \pm 1.8$ & $119.8 \pm 5.5$ \\
\hline 13 & $\left(\mathrm{~A}_{4}\right) \mathrm{C}_{8}$ & $1: 1$ & $9: 1$ & $78.7 \pm 3.6$ & $118.2 \pm 10.7$ \\
\hline 14 & $\mathrm{C}_{8}$ & $1: 2$ & $1: 1$ & $74.8 \pm 3.8$ & $119.2 \pm 9.1$ \\
\hline 15 & $\mathrm{C}_{8}$ & $1: 3$ & $8: 2$ & $91.9 \pm 2.2$ & $123.5 \pm 1.2$ \\
\hline 16 & $\mathrm{C}_{8}$ & $1: 4$ & $7: 3$ & $92.2 \pm 6.4$ & $131.2 \pm 1.2$ \\
\hline
\end{tabular}

SCCPs: short-chain chlorinated paraffins; GC-ECNI-LRMS: gas chromatograph-electron capture negative ion low-resolution mass spectrometry; GC-QTOF-HRMS: gas chromatography-quadrupole time-of-flight high-resolution mass spectrometry. 


\begin{tabular}{|c|c|c|c|c|c|c|}
\hline \multirow[t]{3}{*}{ Levels } & \multicolumn{6}{|c|}{ Factor } \\
\hline & \multicolumn{2}{|c|}{ A } & \multicolumn{2}{|c|}{ B } & \multicolumn{2}{|c|}{ C } \\
\hline & ECNI & QTOF & ECNI & QTOF & ECNI & QTOF \\
\hline $\mathrm{T} 1$ & 80.0 & 117.0 & 82.3 & 119.3 & 82.3 & 119.7 \\
\hline T2 & 81.4 & 117.4 & 75.2 & 115.7 & 72.1 & 106.3 \\
\hline T3 & 74.6 & 112.8 & 82.7 & 118.5 & 80.0 & 120.1 \\
\hline $\mathrm{T} 4$ & 84.4 & 123.0 & 80.2 & 116.8 & 85.9 & 124.1 \\
\hline $\mathrm{R}$ & 9.8 & 10.2 & 7.5 & 3.6 & 13.8 & 17.8 \\
\hline
\end{tabular}

$\mathrm{Tn}$ (T1, T2, T3, T4) is the mean recovery of each factor at the four corresponding levels, and $\mathrm{R}$ is the range of $\mathrm{T} n$.

$\mathrm{A}, \mathrm{B}$, and $\mathrm{C}$ represent the three factors of dispersing sorbent, sample: dispersing sorbent $(\mathrm{m} / \mathrm{m})$ and elution solvent, respectively. ECNI and QTOF represent the two analytical instruments GC-ECNILRMS and GC-QTOF-HRMS, respectively.

The volume of the elution solvent also had a significant impact on the extraction and purification in the MSPD procedure. The optimization of the elution solvent volume adopted the univariate experiments. Four different volumes $(60,80,100$, and $120 \mathrm{~mL})$ of the $7: 3(\mathrm{~V} / \mathrm{V})$ mixture of normal hexane and DCM were used to investigate the effect of elution solvent volume on extraction efficiency. The results are illustrated in Fig. 2. The extraction yield of the target analyte increased along with the increase of elution solvent volume, whereas the matrix-spiked recovery began to decrease when the volume increased to more than $100 \mathrm{~mL}$. This phenomenon may be due to that the excessive volume of elution solvent led to more undesired co-eluted compounds during the extraction process, which can enhance or suppress the

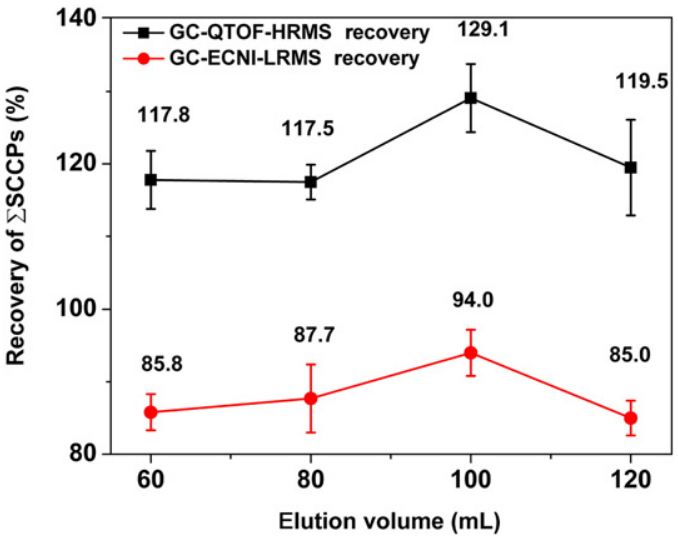

Fig. 2 - Influence of the volume of elution solvent on the recovery of $\Sigma$ SCCPs, $n=3$ replicates.

signal (Gao et al., 2013; Gosetti et al., 2010). So the final elution solvent volume was selected as $100 \mathrm{~mL}$.

\subsection{Lipid removal}

To minimize the subsequent cleanup process after extraction, it is necessary to add co-sorbents into the MSPD glass column below the layer of the sample-dispersing sorbent grinding mixture. Initially, no co-sorbent was added into the column, and oily droplets could be found adhering to the wall of the vial when concentrating the extract to near-dryness with nitrogen gas. The phenomenon confirms the necessity

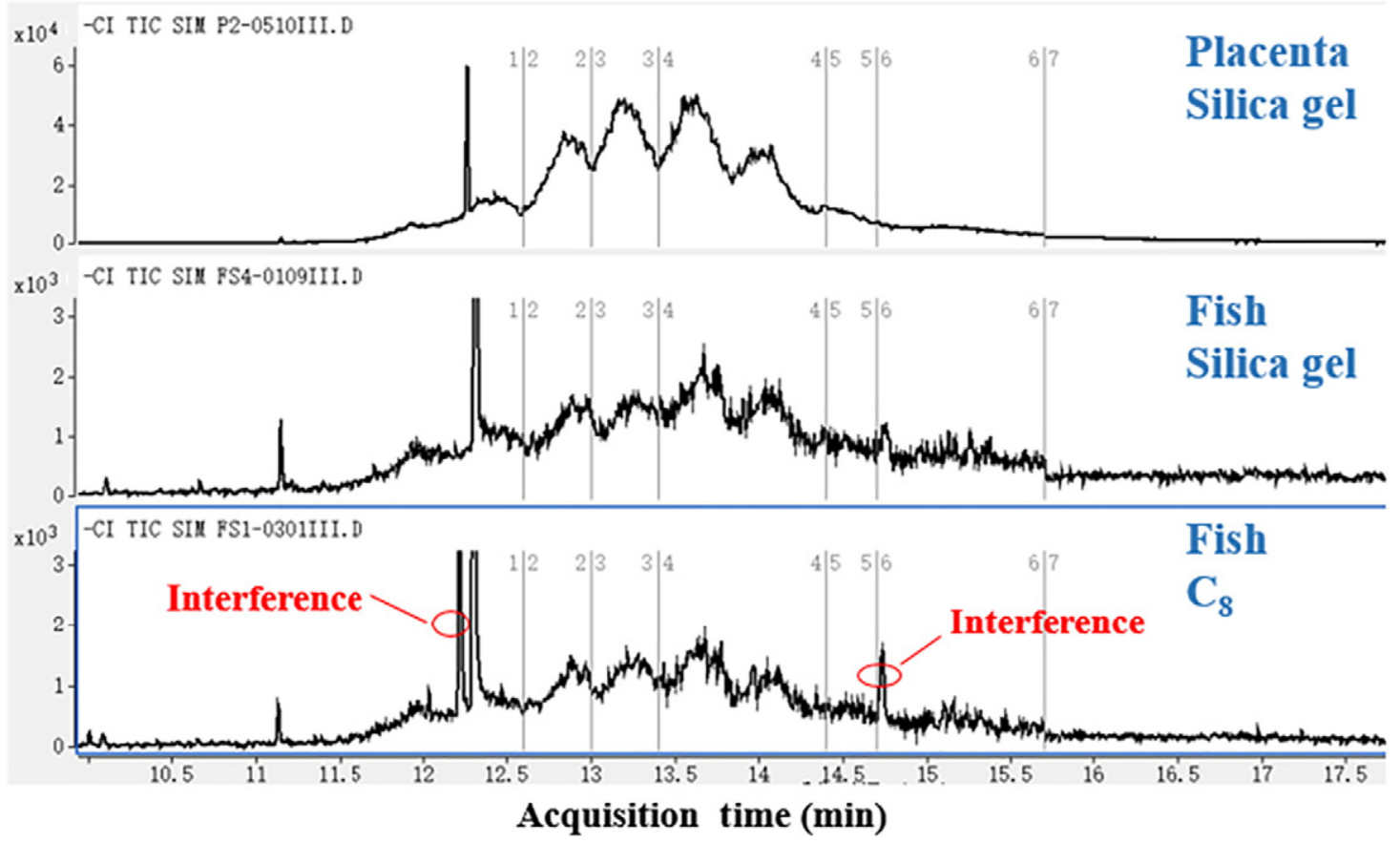

Fig. 1 - The effects of the interference removed in placenta and fish samples using the dispersing sorbent of silica gel and $\mathrm{C}_{8}$, respectively. 
of adding the co-sorbent to remove the lipid from the samples. A previous work indicated that Florisil and acidic silica are effective as fat retainers (Criado et al., 2004). So, in this work, three co-sorbents, $3 \mathrm{~g}$ Florisil, $5 \mathrm{~g}$ acid silica gel (W/W, 44\% $\mathrm{H}_{2} \mathrm{SO}_{4}$ ), and $3 \mathrm{~g}$ Florisil plus $5 \mathrm{~g}$ acid silica gel up the layer of the Florisil, were investigated for their capacity of lipid removal.

The results indicated that only Florisil cannot thoroughly remove the lipid, whereas the acid silica gel and the Florisil plus acid silica gel both can thoroughly remove lipids. In consideration of the cost and eluent velocity, acid silica gel was selected for lipid removal during the pretreatment.

In addition, during the grinding process, anhydrous sodium sulfate was ground with the sample and sorbent together, which not only benefited the dispersal of the placenta tissue due to its large particles but also improved the ability of the sorbent to retain the fats (Pensado et al., 2005).

\subsection{Method validation and the detection limits}

To evaluate the feasibility of the proposed method for different concentration levels of SCCPs, three concentration levels $(200,500,1000 \mathrm{ng})$ of SCCP standards $\left(C_{10}-C_{13}\right.$, chlorine contents of $55.5 \%$ ) were spiked to the fish samples and pretreated under the optimal experimental conditions. Each concentration level was conducted in triplicate, and the non-spiked fish samples were also extracted simultaneously. In addition, the optimized MSPD method was also validated with the human placenta spiked with SCCP standards. Only a relatively low spiking level (200 ng) of SCCP standard $\left(C_{10}-C_{13}\right.$, chlorine content of 55.5\%) was investigated for human placenta because the expected concentrations of SCCPs in the placenta from general population should not be too high. All of the spiked recoveries as well as their corresponding surrogate recoveries are listed in Table 3. For both the fish and placenta samples, high spiked recoveries with good repetition relative standard deviation (RSD) were obtained using GC-ECNI-LRMS and GC-QTOF-HRMS. Moreover, the surrogate standard $\left({ }^{13} \mathrm{C}_{10}-1,5,5,6,6,10\right.$-hexachlorodecane) recoveries were also sufficiently satisfactory with the range of 98.3\%-110.0\% and $111.6 \%-131.1 \%$, by GC-ECNI-LRMS and GCQTOF-HRMS, respectively.

The method detection limit (MDL) is defined as three times the standard deviation of procedural blanks from all of the batches per sample. In this study, the amount of every sample was $1 \mathrm{~g}$ and the MDL for the total SCCPs was 36.8 and $19.2 \mathrm{ng} / \mathrm{g}$
Table 4-Concentrations of total SCCPs in four human placentas $(n=3)$.

\begin{tabular}{|c|c|c|c|c|}
\hline \multirow[t]{2}{*}{ Sample } & \multicolumn{2}{|c|}{$\Sigma$ SCCP (ng/g dw) } & \multicolumn{2}{|c|}{$\begin{array}{l}\text { relative standard } \\
\text { deviation RSD (\%) }\end{array}$} \\
\hline & $\begin{array}{l}\text { GC-ECNI- } \\
\text { LRMS }\end{array}$ & $\begin{array}{l}\text { GC-QTOF- } \\
\text { HRMS }\end{array}$ & $\begin{array}{l}\text { GC-ECNI- } \\
\text { LRMS }\end{array}$ & $\begin{array}{l}\text { GC-QTOF- } \\
\text { HRMS }\end{array}$ \\
\hline P1 & $782.1 \pm 18.2$ & $781.7 \pm 37$ & 2.3 & 4.7 \\
\hline P2 & $743.2 \pm 61.0$ & $756.1 \pm 26.8$ & 8.2 & 3.5 \\
\hline P3 & $<\mathrm{MDL}$ & $<\mathrm{MDL}$ & 62.4 & 30.6 \\
\hline P4 & $422.6 \pm 13.3$ & $436.4 \pm 8.1$ & 3.1 & 1.9 \\
\hline
\end{tabular}

(dw) as measured by GC-ECNI-LRMS and GC-QTOF-HRMS, respectively.

\subsection{SCCPs in human placenta}

The total concentrations of SCCPs in the four human placenta samples are summarized in Table 4. The mean procedural blank value determined by GC-ECNI-LRMS was $80 \mathrm{ng} / \mathrm{g} \mathrm{dw}$, which exceeded $5 \%$ of the concentration of $\Sigma$ SCCPs. The levels of $\Sigma$ SCCPs in the placenta samples are reported on a blankcorrected basis and the concentrations were in the range of $<$ MDL to $782.1 \mathrm{ng} / \mathrm{g}$ by GC-ECNI-LRMS. Meanwhile, the mean procedural blank value determined by GC-QTOF-HRMS was approximately $14 \mathrm{ng} / \mathrm{g}$ dw, which is lower than MDL. Thus, the blank value need not be deducted from the levels of $\Sigma$ SCCPs. The corresponding concentrations of SCCPs in four placenta samples were in the range of $<\mathrm{MDL}$ to $781.7 \mathrm{ng} / \mathrm{g}$, which is consistent with the results obtained by GC-ECNI-LRMS. Compared with GC-ECNI-LRMS, GC-QTOF-HRMS has higher sensitivity and a faster analysis speed but a higher cost.

The distributions of formula groups on the placenta samples were also investigated. Fig. 3 shows that the formula group abundance profiles of the three placenta samples (P1, P2, and P4) showed a similar distribution pattern, and the SCCPs with 7, 8 chlorines were the most predominant congeners, except for P3, which concentration is lower than MDL. The similarity indicates that the exposure sources may be identical among the three pregnant women. To our knowledge, this is the first work reporting the existence of SCCPs in human placenta. Lots of works have proved that general populations might be facing high external and internal exposure levels of SCCPs due to the amounts of production and usage of CP products in China. During development, fetuses have probably been exposed to some

Table 3 - $\Sigma$ SCCPs recovery from the spiked fish and placenta samples and the corresponding surrogate recovery.

\begin{tabular}{|c|c|c|c|c|c|c|c|}
\hline \multirow[t]{2}{*}{ Sample } & \multirow[t]{2}{*}{$\begin{array}{l}\text { Spike level } \\
\text { (ng/g dw) }\end{array}$} & \multicolumn{2}{|c|}{$\begin{array}{l}\text { Mean spiked recovery } \\
(\%)(n=3)\end{array}$} & \multicolumn{2}{|c|}{$\begin{array}{l}\text { Surrogate recovery } \\
\qquad(\%)(n=6)\end{array}$} & \multicolumn{2}{|c|}{$\begin{array}{c}\text { relative standard } \\
\text { deviation (RSD) } \\
(\%)\end{array}$} \\
\hline & & ECNI & QTOF & ECNI & QTOF & ECNI & QTOF \\
\hline Fish & 200 & 89.7 & 100.4 & 98.3 & 119.0 & $9.5(2.8)$ & $6.6(6.1)$ \\
\hline Fish & 500 & 86.3 & 111.8 & 98.2 & 118.0 & $2.5(15.0)$ & $2.8(8.6)$ \\
\hline Fish & 1000 & 94.0 & 129.1 & 110.0 & 116.6 & $3.4(3.4)$ & $3.6(5.7)$ \\
\hline Placenta & 200 & 77.4 & 91.4 & 106.6 & 131.1 & $10.2(2.4)$ & $5.6(7.4)$ \\
\hline
\end{tabular}

The numbers in brackets are the RSD of the surrogate recoveries.

ECNI and QTOF represent the two analytical instruments GC-ECNI-LRMS and GC-QTOF-HRMS, respectively. 

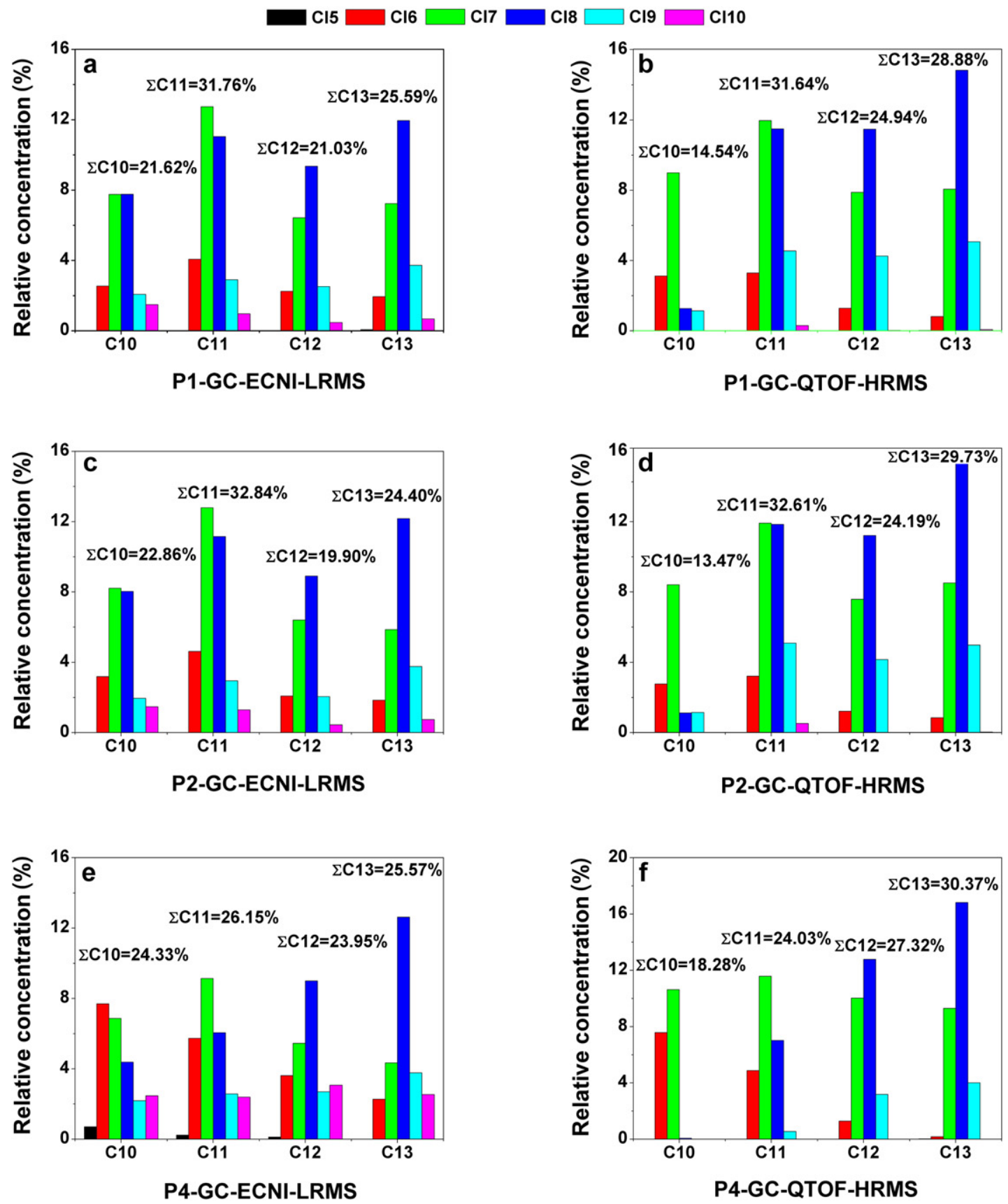

Fig. 3 - Different formula group abundances (in percentages) of SCCPs in different human placenta samples (P1, P2, P4) obtained using GC-ECNI-LRMS and GC-QTOF-HRMS.

toxic substances, especially POPs. Thus, monitoring the level of SCCPs in human placenta is critical to evaluate the health risk in the uterine life period by transplacental transfer.

\section{Conclusions}

In this work, an efficient, reliable and rapid pretreatment method based on MSPD for SCCPs in human placenta was firstly developed and validated using GC-ECNI-LRMS and GC-
QTOF-HRMS. The MSPD method using silica gel as a sorbent and a mixture of hexane and DCM $(7: 3, \mathrm{~V} / \mathrm{V})$ as the elution allowed for the extraction of the analytes and the simultaneous removal of lipids from placenta samples. The high recoveries with good repeatability in human placenta indicated that the developed method for SCCPs in human placenta can be used for the large scale investigation on CP exposure to the general population. It also has reference meaning for extracting other POPs in human placenta samples. Although only four placenta samples were investigated, three of them 
were found to have SCCPs, indicating the urgency of survey of human exposure sources, the transplacental transfer of CPs of mothers and fetus, etc.

\section{Acknowledgments}

This work was supported by the National Natural Science Foundation of China (Nos. 21625702, 21337002, 21621064), the National Basic Research Program of China (No. 2015CB453102), and the Strategic Priority Research Program of the Chinese Academy of Science (No. XDB14010400) for the joint financial support.

\section{R E F E R E N C E S}

Barber, J.L., Sweetman, A.J., Thomas, G.O., Braekevelt, E., Stern, G.A., Jones, K.C., 2005. Spatial and temporal variability in air concentrations of short-chain (C10-C13) and medium-chain (C14-C17) chlorinated n-alkanes measured in the UK atmosphere. Environ. Sci. Technol. 39, 4407-4415.

Barker, S.A., 2000. Matrix solid-phase dispersion. J. Chromatogr. A 885, 115-127.

Ben, Y.J., Li, X.H., Yang, Y.L., Li, L., Zheng, M.Y., Wang, W.Y., Xu, X.B., 2014. Placental transfer of dechlorane plus in mother-infant pairs in an E-waste recycling area (Wenling, China). Environ. Sci. Technol. 48, 5187-5193.

Bennie, D., Sullivan, C., Maguire, R., 2000. Occurrence of chlorinated paraffins in beluga whales (Delphiapterus leucas) from the St. Lawrence River and rainbow trout (Oncorhynchus mykiss) and carp (Cyprinus carpio) from Lake Ontario. Water Qual. Res. J. Can. 35, 263-281.

Bettina, H., Hermann, F., Wolfgang, V.L., Mehmet, C., 2011. Effects of chain length, chlorination degree, and structure on the octanol-water partition coefficients of polychlorinated n-alkanes. Environ. Sci. Technol. 45, 2842-2849.

Campbell, I., McConnell, G., 1980. Chlorinated paraffins and the environment. 1. Environmental occurrence. Environ. Sci. Technol. 14, 1209-1214.

Capriotti, A.L., Cavaliere, C., Giansanti, P., Gubbiotti, R., Samperi, R., Laganà, A., 2010. Recent developments in matrix solid-phase dispersion extraction. J. Chromatogr. A 1217, 2521-2532.

Carro, A., Lorenzo, R., Fernández, F., Rodil, R., Cela, R., 2005. Multi-residue screening of chlorinated and brominated compounds from aquaculture samples using matrix solid-phase dispersion-gas chromatography-mass spectrometry. J. Chromatogr. A 1071, 93-98.

Chen, M.Y., Luo, X.J., Zhang, X.L., He, M.J., Chen, S.J., Mai, B.X., 2011. Chlorinated paraffins in sediments from the Pearl River Delta, South China: spatial and temporal distributions and implication for processes. Environ. Sci. Technol. 45, 9936-9943.

Cheng, J.P., Fujimura, M., Bo, D., 2015. Assessing pre/post-weaning neurobehavioral development for perinatal exposure to low doses of methylmercury. J. Environ. Sci. 38, 36-41.

Coelhan, M., 2010. Levels of chlorinated paraffins in water. Clean Soil Air Water 38, 452-456.

Criado, M.R., Fernández, D.H., Pereiro, I.R., Torrijos, R.C., 2004. Application of matrix solid-phase dispersion to the determination of polychlorinated biphenyls in fat by gas chromatography with electron-capture and mass spectrometric detection. J. Chromatogr. A 1056, 187-194.

Dewailly, E., Bruneau, S., Ayotte, P., Laliberte, C., Gingras, S., Belanger, D., et al., 1993. Health status at birth of Inuit newborn prenatally exposed to organochlorines. Chemosphere 27, 359-366.

Fridén, U.E., McLachlan, M.S., Berger, U., 2011. Chlorinated paraffins in indoor air and dust: concentrations, congener patterns, and human exposure. Environ. Int. 37, 1169-1174.

Gao, Y., Zhang, H.J., Su, F., Tian, Y.Z., Chen, J.P., 2012. Environmental occurrence and distribution of short chain chlorinated paraffins in sediments and soils from the Liaohe River Basin, PR China. Environ. Sci. Technol. 46, 3771-3778.

Gao, Y., Sun, Y., Wang, Y.P., Zhang, J., Xu, B., Zhang, H.Q., et al., 2013. A practical and rapid method for the simultaneous isolation, purification and quantification of geniposide from the fruit of Gardenia jasminoides Ellis by MSPD extraction and UFLC analysis. Anal. Methods 5, 4112-4118.

Gao, W., Wu, J., Wang, Y.W., Jiang, G.B., 2016. Quantification of short-and medium-chain chlorinated paraffins in environmental samples by gas chromatography quadrupole time-of-flight mass spectrometry. J. Chromatogr. A 1452, 98-106.

Gosetti, F., Mazzucco, E., Zampieri, D., Gennaro, M.C., 2010. Signal suppression/enhancement in high-performance liquid chromatography tandem mass spectrometry. J. Chromatogr. A 1217, 3929-3937.

Harada, K.H., Takasuga, T., Hitomi, T., Wang, P., Matsukami, H., Koizumi, A., 2011. Dietary exposure to short-chain chlorinated paraffins has increased in Beijing, China. Environ. Sci. Technol. 45, 7019-7027.

Huel, G., Godin, J., Frery, N., Girard, F., Moreau, T., Nessmann, C., Blot, P., 1992. Aryl hydrocarbon hydroxylase activity in human placenta and threatened preterm delivery. J. Expo. Anal. Environ. Epidemiol. 3, 187-199.

Iino, F., Takasuga, T., Senthilkumar, K., Nakamura, N., Nakanishi, J., 2005. Risk assessment of short-chain chlorinated paraffins in Japan based on the first market basket study and species sensitivity distributions. Environ. Sci. Technol. 39, 859-866.

Jansson, B., Andersson, R., Asplund, L., Litzen, K., Nylund, K., Sellströom, U., et al., 1993. Chlorinated and brominated persistent organic compounds in biological samples from the environment. Environ. Toxicol. Chem. 12, 1163-1174.

Kristenson, E.M., Udo, A.T., Ramos, L., 2006. Recent advances in matrix solid-phase dispersion. TrAC Trends Anal. Chem. 25, 96-111.

Li, N.Q., Wania, F., Lei, Y.D., Daly, G.L., 2003. A comprehensive and critical compilation, evaluation, and selection of physical-chemical property data for selected polychlorinated biphenyls. J. Phys. Chem. Ref. Data 32, 1545-1590.

Liu, Q., Shi, J.B., Sun, J.T., Wang, T., Zeng, L.X., Zhu, N.L., Jiang, G.B., 2011. Graphene-assisted matrix solid-phase dispersion for extraction of polybrominated diphenyl ethers and their methoxylated and hydroxylated analogs from environmental samples. Anal. Chim. Acta 708, 61-68.

Main, K.M., Kiviranta, H., Virtanen, H.E., Sundqvist, E., Tuomisto, J.T., Tuomisto, J., et al., 2007. Flame retardants in placenta and breast milk and cryptorchidism in newborn boys. Environ. Health Perspect. 1519-1526.

Myren, M., Mose, T., Mathiesen, L., Knudsen, L.E., 2007. The human placenta-an alternative for studying foetal exposure. Toxicol. in Vitro 21, 1332-1340.

Pensado, L., Casais, M., Mejuto, M., Cela, R., 2005. Application of matrix solid-phase dispersion in the analysis of priority polycyclic aromatic hydrocarbons in fish samples. J. Chromatogr. A 1077, 103-109.

Reth, M., Oehme, M., 2004. Limitations of low resolution mass spectrometry in the electron capture negative ionization mode for the analysis of short- and medium-chain chlorinated paraffins. Anal. Bioanal. Chem. 378, 1741-1747. 
Reth, M., Zencak, Z., Oehme, M., 2005. New quantification procedure for the analysis of chlorinated paraffins using electron capture negative ionization mass spectrometry. J. Chromatogr. A 1081, 225-231.

Thomas, G.O., Farrar, D., Braekevelt, E., Stern, G., Kalantzi, O.I., Martin, F.L., et al., 2006. Short and medium chain length chlorinated paraffins in UK human milk fat. Environ. Int. 32, 34-40.

Tomy, G.T., Muir, D.C., Stern, G.A., Westmore, J.B., 2000. Levels of C10-C13 polychloro-n-alkanes in marine mammals from the Arctic and the St. Lawrence River estuary. Environ. Sci. Technol. 34, 1615-1619.

UNEP/POPRC.11/10/Add.2, 2015. Risk profile: short-chained chlorinated paraffins. http://chm.pops.int/TheConvention/ POPsReviewCommittee/Meetings/POPRC11/Overview/tabid/ 4558/mctl/ViewDetails/EventModID/871/EventID/553/xmid/ 13837/Default.aspx.

Valsamaki, V., Boti, V., Sakkas, V., Albanis, T.A., 2006. Determination of organochlorine pesticides and polychlorinated biphenyls in chicken eggs by matrix solid phase dispersion. Anal. Chim. Acta 573, 195-201.
Wang, T., Han, S.L., Yuan, B., Zeng, L.X., Li, Y.M., Wang, Y.W., et al., 2012. Summer-winter concentrations and gas-particle partitioning of short chain chlorinated paraffins in the atmosphere of an urban setting. Environ. Pollut. 171, 38-45.

Wang, Y., Li, J., Cheng, Z.N., Li, Q.L., Pan, X.H., Zhang, R.J., et al., 2013. Short-and medium-chain chlorinated paraffins in air and soil of subtropical terrestrial environment in the Pearl River Delta, South China: distribution, composition, atmospheric deposition fluxes, and environmental fate. Environ. Sci. Technol. 47, 2679-2687.

Zeng, L.X., Wang, T., Han, W.Y., Yuan, B., Liu, Q., Wang, Y.W., et al., 2011. Spatial and vertical distribution of short chain chlorinated paraffins in soils from wastewater irrigated farmlands. Environ. Sci. Technol. 45, 2100-2106.

Zeng, L.X., Li, H.J., Wang, T., Gao, Y., Xiao, K., Du, Y.G., et al., 2013. Behavior, fate, and mass loading of short chain chlorinated paraffins in an advanced municipal sewage treatment plant. Environ. Sci. Technol. 47, 732-740. 